\title{
Comparing the Efficacy of a Selective Cyclooxygenase-2 Inhibitor and an Anticholinergic in Patients with Benign Prostatic Hyperplasia and Refractory Nocturia
}

\author{
Min Seek Lim, M.D., Choal Hee Park, M.D., Hyuk Soo Chang, M.D., Chun Il Kim, M.D. \\ Department of Urology, Keimyung University School of Medicine, \\ Daegu, Korea
}

\begin{abstract}
Nocturia is one of the most bothersome symptoms of benign prostatic hyperplasia (BPH) that can cause deteriorate quality of life. We compared the efficacy of a selective cyclooxygenase (COX)-2 inhibitor (meloxicam) with anticholinergic (propiverine $\mathrm{HCl}$ ) for the patients with BPH who have complained of refractory nocturia. Sixty BPH patients with two or more episodes of nocturia, despite standard medication, were enrolled in this prospective study. The patients were randomized to receive either a single meloxicam $7.5 \mathrm{mg}$ (32 patients) or a propiverine $\mathrm{HCl} 20 \mathrm{mg}$ (28 patients) before sleep for 2 weeks. The efficacy assessments were based on data in the patients' frequency-volume chart for three days before and after the treatment. Results were compared between the selective COX-2 inhibitor group and the anticholinergic group. The frequency of nocturia decreased $-1.7 \pm 0.8(\mathrm{p}=0.000)$ and $-0.9 \pm$ $0.9(p=0.000)$ episodes after 2 weeks in the selective COX -2 inhibitor and the anticholinergic groups, respectively. The nocturnal urine volume was decreased $-163.6 \pm 194.9 \mathrm{~mL}$ in the selective COX -2 inhibitor group $(\mathrm{p}=0.000)$ and $-9.4 \pm 45.2 \mathrm{~mL}$ in the anticholinergic group $(p=0.281)$. At daytime, however frequency and urine volume did not show any differences between both groups. The nocturnal functional bladder capacity was significantly increased from $223.2 \pm 41.5 \mathrm{~mL}$ to $269.6 \pm 48.5 \mathrm{~mL}$ in anticholinergic group $(\mathrm{p}=0.000)$, but not for the selective COX-2 inhibitor group (from $254.4 \pm 39.4 \mathrm{~mL}$ to $261.5 \pm 48.6 \mathrm{~mL}, \mathrm{p}=0.281$ ). In conclusion, selective COX-2 inhibitor is a more effective treatment than anticholinergic for the treatment of refractory nocturia in patients with $\mathrm{BPH}$. The main mechanism of $\mathrm{COX}-2$ inhibitor for decreasing nocturia may be the reduction of nocturnal urine production. The anticholinergic also can reduce nocturia by increasing nocturnal functional bladder capacity.
\end{abstract}

Key Words : Cholinergic antagonist, Cyclooxygenase 2 inhibitor, Nocturia, Prostatic hyperplasia

Corresponding Author: Chun Il Kim, M.D., Department of Urology, Keimyung University School of Medicine 216, Dalseongno, Jung - gu, Daegu, 700-712 KOREA

Tel: +82-53-250-7646 E-mail: cikim@dsmc.or.kr 


\section{Introduction}

Nocturia is a major problem in the patients with lower urinary tract symptoms (LUTS) and results in a deteriorated quality of life for patients with benign prostatic hyperplasia (BPH). The standard treatments for BPH such as alpha blockers or 5-alpha reductase inhibitors (5-ARI) have treated nocturia, but they are insufficient. Even if these drugs are used, nocturia reduced by $50 \%$ or more in only $25-39 \%$ of cases [1]. Even in a study that had a follow-up over 4 years, nocturia improved by 1 or more times in only $42-47 \%$ of case [2]. Although antidiuretics are effective against nocturia, there is concern about their long-term safety for elder patients $[3,4]$.

Recently, a number of studies have suggested that non-steroidal antiinflammatory drugs (NSAIDs) are effective against nocturia [5-7]. Some studies have suggested that selective cyclooxygenase (COX) -2 inhibitors were an effective way of treating refractory nocturia in patients with BPH [8]. However, other studies suggested that anticholinergics are an effective treatment for nocturia $[9,10]$. However there has been no report comparing the efficacy of NSAIDs and anticholinergics in treating nocturia. So we performed this study to compare the efficacy of a selective COX-2 inhibitor with an anticholinergic in patients with BPH who suffered from refractory nocturia despite their standard $\mathrm{BPH}$ medication.

\section{Materials and Methods}

A study for 60 patients with BPH was conducted from February to December of 2008. All the patients had used the alpha blocker alone or alpha blocker with 5-ARI for more than six months, but still complained 2 or more episodes of nocturia. Exclusion criteria were patients with urinary tract infection, prostate or bladder tumor, urethral stricture, neurogenic bladder, gastrointestinal ulcer and bleeding or severe concomitant condition threatening life; who underwent urethral reconstructive surgery, prostatectomy or non-medical treatment for prostate; or hypersensitive to NSAIDs.

The patients were randomly divided into selective COX-2 inhibitor (COX-2I) group $(\mathrm{n}=32)$ and anticholinergic (AC) group $(n=28)$ and each group is treated with meloxicam $7.5 \mathrm{mg}$ or propiverine $\mathrm{HCl} 20 \mathrm{mg}$ once every day before sleep for 2 weeks, respectively. All the patients were instructed to fill out International Prostate Symptom Score (IPSS) and keep 3-day frequencyvolume chart before and after the treatment.

Frequency of nocturia, nocturnal urine volume, daytime frequency, daytime urine volume, nocturnal functional bladder capacity (FBC) and IPSS were compared between both groups. Also, the adverse effects were recorded. Statistical analysis was performed by using $t$-test and considered significant at $\mathrm{P}<0.05$.

\section{Results}

Mean ages were 68.8 and 65.0 years old in the COX-2I group and the AC group, 
respectively. Previous medical treatments for BPH were alpha blocker alone in 20 and 18 patients, and combination treatment with alpha blocker and 5-ARI in 12 and 10 patients in both groups, respectively. There was no difference in baseline IPSS and parameters in voiding diary in two groups.

Of 32 patients in the COX-2I group, 14 patients $(43.8 \%)$ experienced a reduction in nocturia by two or more episodes, and 11 patients (34.3\%) experienced 1 2 episodes of reduction. And 7 patients (21.9\%) showed less than an episode of reduction. No subjects experienced increased nocturia. Of 28 patients in the AC group, 4 patients (14.3\%) experienced a reduction in nocturia by two or more episodes, and 7 patients $(25.0 \%)$ experienced 1 2 episodes of reduction. And 17 patients (60.7\%) showed less than an episode of reduction. No patients experienced a reduction in nocturia by three or more episodes or increased nocturia (Table 1).

Table 2 shows the results of 2 -week treatment in frequency-volume chart parameters. The frequency of nocturia decreased from $3.9 \pm 1.0$ to $2.2 \pm 0.5$ $(\mathrm{p}=0.000)$ and from $3.6 \pm 0.9$ to $2.7 \pm 0.4$ $(p=0.000)$ in the COX-2I group and the AC group, respectively. The COX-2I group showed more reduction of nocturia than the $\mathrm{AC}$ group $(-1.7 \pm 0.8$ vs $-0.9 \pm 0.9)$ $(\mathrm{p}=0.000)$.

Changes of nocturnal urine volumes were $-163.6 \pm 194.9 \mathrm{~mL}$ and $-9.4 \pm 45.2 \mathrm{~mL}$ in both groups, respectively. The nocturnal urine volume decreased significantly in the COX-2I group $(p=0.000)$, but not significantly in the $\mathrm{AC}$ group.

No difference was found in the changes of daytime frequency between both groups, and baseline and after 2-week treatment in each group ( $p=0.089$ and 0.752 in the COX-2I group and the $\mathrm{AC}$ group, respectively). The daytime urine volume increased by $99.4 \pm$ $255.3 \mathrm{~mL}$ in the COX-2I group ( $\mathrm{p}=0.035)$ and $44.0 \pm 293.8 \mathrm{~mL}$ in the AC group ( $>>0.05)$. But there was no difference between both groups.

The nocturnal and daytime $\mathrm{FBC}$ were significantly increased in the ACs group $(p=0.000)$. But the COX-2I group showed no differences in nocturnal and daytime FBC between baseline and after 2 weeks of treatment.

Table 1. Changes in number of nocturia in selective COX-2 inhibitor (COX-2I) and anticholinergic (AC) group after 2 weeks of treatment

\begin{tabular}{lcc}
\hline Frequency change of nocturia & COX-2I group & AC group \\
\hline decrease $\geq 3$ & $2(6.3 \%)$ & 0 \\
$2 \leq$ decrease $<3$ & $12(37.5 \%)$ & $4(14.3 \%)$ \\
$1 \leq$ decrease $<2$ & $11(34.3 \%)$ & $7(25.0 \%)$ \\
decrease $<1$ & $7(21.9 \%)$ & $17(60.7 \%)$ \\
Increase & 0 & 0 \\
\hline Total & $32(100 \%)$ & $28(100 \%)$ \\
\hline
\end{tabular}


Table 2. Changes from baseline to 2 weeks of treatment in frequency-volume chart parameters for COX-2I and $\mathrm{AC}$ group

\begin{tabular}{|c|c|c|c|}
\hline & COX-2I group & $\mathrm{AC}$ group & $P$-value \\
\hline \multicolumn{4}{|l|}{ Nocturia (No.) } \\
\hline Baseline & $3.9 \pm 1.0$ & $3.6 \pm 0.9$ & 0.170 \\
\hline 2-wk treatment & $2.2 \pm 0.5^{* *}$ & $2.7 \pm 0.4^{* *}$ & 0.000 \\
\hline Difference & $-1.7 \pm 0.8$ & $-0.9 \pm 0.9$ & 0.000 \\
\hline \multicolumn{4}{|c|}{ Nocturnal urine volume $(\mathrm{mL})$} \\
\hline Baseline & $729.0 \pm 204.9$ & $572.0 \pm 195.1$ & 0.004 \\
\hline 2-wk treatment & $565.4 \pm 222.4^{* *}$ & $562.6 \pm 178.9$ & 0.957 \\
\hline Difference & $-163.6 \pm 194.9$ & $-9.4 \pm 45.2$ & 0.000 \\
\hline \multicolumn{4}{|c|}{ Daytime frequency (No.) } \\
\hline Baseline & $9.3 \pm 2.4$ & $9.4 \pm 1.4$ & 0.777 \\
\hline 2-wk treatment & $9.8 \pm 1.9$ & $9.3 \pm 2.4$ & 0.355 \\
\hline Difference & $0.6 \pm 1.8$ & $-0.1 \pm 1.8$ & 0.156 \\
\hline \multicolumn{4}{|c|}{ Daytime urine volume $(\mathrm{mL})$} \\
\hline Baseline & $1659.7 \pm 472.3$ & $1728.9 \pm 316.4$ & 0.514 \\
\hline 2-wk treatment & $1759.1 \pm 486.8^{*}$ & $1772.8 \pm 304.2$ & 0.898 \\
\hline Difference & $99.4 \pm 255.3$ & $44.0 \pm 293.8$ & 0.438 \\
\hline \multicolumn{4}{|c|}{ Nocturnal functional bladder capacity $(\mathrm{mL})$} \\
\hline Baseline & $254.4 \pm 39.4$ & $223.2 \pm 41.5$ & 0.004 \\
\hline 2-wk treatment & $261.5 \pm 48.6$ & $269.6 \pm 48.5^{* *}$ & 0.521 \\
\hline Difference & $7.1 \pm 36.5$ & $46.4 \pm 41.4$ & 0.000 \\
\hline \multicolumn{4}{|c|}{ Daytime functional bladder capacity $(\mathrm{mL})$} \\
\hline Baseline & $282.7 \pm 50.3$ & $268.0 \pm 68.8$ & 0.344 \\
\hline 2-wk treatment & $292.2 \pm 45.2$ & $320.6 \pm 37.2 * *$ & 0.011 \\
\hline Difference & $9.5 \pm 37.2$ & $52.6 \pm 66.8$ & 0.003 \\
\hline
\end{tabular}

$* \mathrm{p}<0.05, * * \mathrm{p}<0.01$ comparing between baseline and 2 -wk treatment.

Table 3 shows the changes of IPSS after 2 weeks of treatment. Total IPSS decreased by $-2.0 \pm 4.3(p=0.014)$ and $-0.7 \pm 1.5$ $(p=0.019)$ in the COX-2I group and the AC group, respectively. However, there was no difference between two groups $(p=0.141)$. 
Table 3. Changes from baseline to 2 week-treatment in International Prostate Symptom Score for COX-2I and $\mathrm{AC}$ group

\begin{tabular}{|c|c|c|c|}
\hline & COX-2I group & AC group & $P$-value \\
\hline \multicolumn{4}{|l|}{ Total } \\
\hline Baseline & $20.4 \pm 4.8$ & $20.7 \pm 5.7$ & 0.803 \\
\hline 2-wk treatment & $18.4 \pm 5.9^{*}$ & $20.0 \pm 4.6^{*}$ & 0.241 \\
\hline Difference & $-2.0 \pm 4.3$ & $-0.7 \pm 1.5$ & 0.141 \\
\hline \multicolumn{4}{|l|}{ Voiding } \\
\hline Baseline & $11.8 \pm 4.3$ & $12.3 \pm 3.6$ & 0.603 \\
\hline 2-wk treatment & $11.3 \pm 4.7$ & $8.4 \pm 3.2$ & 0.254 \\
\hline Difference & $-0.5 \pm 2.0$ & $0.2 \pm 0.5$ & 0.074 \\
\hline \multicolumn{4}{|l|}{ Storage } \\
\hline Baseline & $8.6 \pm 2.9$ & $8.4 \pm 3.2$ & 0.835 \\
\hline 2-wk treatment & $7.1 \pm 2.6^{* *}$ & $7.6 \pm 3.0^{* * *}$ & 0.478 \\
\hline Difference & $-1.5 \pm 2.9$ & $-0.9 \pm 1.3$ & 0.259 \\
\hline \multicolumn{4}{|l|}{ Quality of life } \\
\hline Baseline & $3.5 \pm 0.7$ & $3.1 \pm 1.0$ & 0.055 \\
\hline 2-wk treatment & $3.7 \pm 0.6^{* *}$ & $2.8 \pm 0.8^{* *}$ & 0.100 \\
\hline Difference & $-0.4 \pm 0.5$ & $-0.3 \pm 0.5$ & 0.365 \\
\hline
\end{tabular}

$* \mathrm{p}<0.05, * * \mathrm{p}<0.01$ comparing between baseline and 2 -wk treatment.

Storage symptom ( $p=0.005$ and 0.001 , respectively) and quality of life $(p=0.000$ and 0.001 , respectively) also more decreased in the COX-2I group than the AC group. But voiding symptom scores shows no difference after 2 weeks of treatment in two groups ( $p=0.143$ and 0.096 , respectively).

Serious adverse events did not occurr during this study. Mild adverse events were occurred in $8(25.0 \%)$ and 9 patients $(32.1 \%)$ in the COX-2I and the AC group, respectively (Table 4).

\section{Discussion}

It has been reported that $28 \%$ of adults 30 years or older complain of nocturia, and the 
Table 4. Treatment-emergent adverse events in COX-2I and $\mathrm{AC}$ groups

\begin{tabular}{llc}
\hline & Adverse events & $\begin{array}{c}\text { No. of } \\
\text { patients (\%) }\end{array}$ \\
\hline \multirow{2}{*}{ COX-2I group } & Leg edema & $3(9.4)$ \\
& Dizziness & $2(6.3)$ \\
& Gastric discomfort & $3(9.4)$ \\
AC group & Dry mouth & $7(25.0)$ \\
& Headache & $2(7.1)$ \\
\hline
\end{tabular}

ratio increases proportionally with aging $[11,12]$. Some reports give nocturia as the most common reason for sleep disturbance in 60 80 years old [13].

Many patients having BPH are suffering from nocturia. In many cases, however, treatments for BPH are not effective against nocturia. Generally, adults need sleep about 7 8 hours a day to recover mentally and physically. Insufficient sleeping due to nocturia causes daytime sleepiness, hindering daily life and possibly causing physical or mental impairment. It has been documented that two or more episodes of nocturia considerably affect quality of life and increase the risk of falling and bruising in older people $[12,14]$.

Various possible causes of nocturia have been suggested, including morbid conditions such as cardiovascular diseases, diabetes, anxiety, sleep disorder and environmental factors. Suggested concrete causes include disorders related to the control of urine production during both daytime and nighttime, nocturnal polyuria, decreased bladder capacity including overactive bladder, increased sodium excretion and functional disorders of renal tubules due to lower tract obstructions [9,15,16]. Kirkland et al. [17] compared the volume of urine produced in a 24 -hour period and the daytime/nighttime ratio of urine production between younger and elder groups. They reported that urine productions in a 24hour period were 1,611 $\mathrm{mL}$ and 1,652 $\mathrm{mL}$ in two groups, respectively. Thus, there was no significant difference. However, the ratios of daytime and nocturnal urine production of the younger and older groups were 2.07 and 1.08, indicating as significant difference interms of increased nocturnal urine production for elder people. This is thought to be caused by changes in glomerular filtration rates, the functioning of renal tubules and cardiac functions.

Desmopressin is an antidiuretic used to treat nocturnal polyuria. However, its use may cause serious complications such as hyponatremia. Bae et al. [3] reported that desmopressin was effective in reducing the urine volume, but the level of serum sodium significantly decreased from $142.2 \pm 1.9$ $\mathrm{mmol} / \mathrm{L}$ to $136.0 \pm 4.27 \mathrm{mmol} / \mathrm{L} 12$ months after desmopressin treatment. Rembratt et al. [4] analyzed risk factors for hyponatremia in patients who received desmopressin. Hyponatremia measuring $134 \mathrm{mmol} / \mathrm{L}$ or less was observed in $15 \%$, and serious hyponatremia measuring less than 130 $\mathrm{mmol} / \mathrm{L}$ was observed in $3 \%$. They also reported that the age of the patients was the most important risk factor for hyponatremia and that patients 65 years or older with a basal serum sodium value lower than the normal range had an incidence rate of $75 \%$ for serious hyponatremia. Given the seresults, the risk of hyponatremia must not be 
overlooked when giving desmopressin to older patients and serum sodium levels must be periodically checked.

Decreased bladder capacity is also recognized as an important cause of nocturia. It is acknowledged that anticholinergics act on the muscarinic receptors in the detrusor muscles. Anticholinergics suppress the involuntary activity of myocytes during storage periods, thereby reducing the hyperactivity of the detrusor muscles. Brubaker and FitzGerald [9] reported that solifenacin decreased nocturia by -0.6 episode for the patients without nocturnal polyuria. Fitzgerald et al. [10] reported that nocturia significantly decreased by $-0.28 \pm$ 1.15 episodes after 8 weeks of treatment. In the present study, nocturia decreased by -0.9 \pm 0.9 episodes for the patients with anticholinergics. However, the anticholinergics may cause adverse events such as dry mouth, constipation or dysopsia. Jünemann et al. [18] used propiverine $\mathrm{HCl}$ for patients with overactive bladders and reported the adverse effects of dry mouth in $22 \%$, constipation in $3 \%$, headache in $2 \%$, and visual disturbance in $5 \%$ of the patients. In the present study, similar adverse effects occurred, but there was no patients for whom the drug was stopped.

COX an enzyme converting arachidonic acid into prostaglandins (PGs) and other eicosanoids, has two sub-types, COX-1 and COX-2. The physiologic actions of prostaglandin E2 (PGE2) and prostacyclin are to maintain renal blood flow and glomerular filtration rates, to suppress tubular sodium re-absorption, to counteract anti-diuretic hormones and to regulate renin secretion [19]. They also reduce aldosterone secretion and increase the excretion of water and sodium through glomerular capillaries, thereby inducing diuretic action. NSAIDs suppress the generation of COX to counteract PGs. Several studies have reported that the effects of NSAIDs on nocturia were 74 to $83 \%$, which is comparable with that of present study $[5,7,8]$. Based on these studies, it can be assumed that the effect of NSAIDs on nocturia is due to reduced urine production.

It is unknown which COX inhibitor subtype affects nocturia. Falahatkar et al. [8] reported that celecoxib, a selective COX -2 inhibitor, decreased nocturia in $83 \%$. Unfortunately, frequency-volume chart was not recorded in the study, thus, the effect of selective COX-2 inhibitors on nocturia could not be determined.

Based on the results of the present study, the selective $\mathrm{COX}-2$ inhibitor and anticholinergic are effective for the patients with BPH and nocturia. Selective COX-2 inhibitor decreased nocturia by reducing nocturnal urine production, and anticholinergic decreased nocturia by increasing nocturnal FBC. However, it was demonstrated that selective COX-2 inhibitors were more effective compared to anticholinergics.

There was no serious adverse effects of selective COX -2 inhibitors and the adverse effects were less frequent compared to anticholinergics. Also, unlike desmopressin, the use of selective COX-2 inhibitors in elder patients need not be limited, nor are periodic blood tests required.

\section{Summary}

Selective COX - 2 inhibitors can reduce nocturia more effectively than anticholinergics. 
The selective COX -2 inhibitor taken before sleep reduces nighttime urine production, thereby reducing nocturia. However, it does not affect daytime voiding amounts. It can also improve IPSS and quality of life scores. The selective COX -2 inhibitors are considered to be safe for use in older patients. However, this study, involved small groups and was conducted over a short period of time. Therefore, large-scale, long-term studies are necessary in the future. Studies to compare the effects of different NSAIDs based on COX selectivity on nocturia are also necessary.

\section{References}

1. Johnson TM, Jones K, Williford WO, Kutner MH, Issa MM, Lepor $\mathrm{H}$. Changes in nocturia from medical treatment of benign prostatic hyperplasia: secondary analysis of the department of Veterans Affairs Cooperative Study Trial. J Urol 2003;170:145-8.

2. Johnson TM, Burrows PK, Kusek JW, Nyberg LM, Tenover JL, Lepor H, et al. The effect of doxazosin, finasteride and combination therapy on nocturia in men with benign prostatic hyperplasia. J Urol 2007;178:2045-51.

3. Bae JH, Oh MM, Shim KS, Cheon J, Lee JG, Kim JJ, et al. The effects of long-term administration of oral desmopressin on the baseline secretion of antidiuretic hormone and serum soduim concentration for the treatment of nocturia: a circadian study. J Urol 2007;178:200-3.

4. Rembratt A, Riis A, Norgaard JP. Desmopressin treatment in nocturia; an analysis of risk factors for hyponatremia. Neurourol Urodyn 2006;25:105-9.

5. Araki T, Yokoyama T, Kumon H. Effectiveness of a nonsteroidal anti-inflammatory drugs for nocturia on patients with benign prostatic hyperplasia: a prospective non-randomized study of loxoprofen sodium $60 \mathrm{mg}$ once daily before sleeping. Acta Med Okayama 2004;58:45-9.

6. Saito M, Kawatani M, Kinoshita Y. Effectiveness of an anti-inflammatory drug, loxoprofen, for patients with nocturia. Int J Urol 2005;12:779-82.

7. Okada S, Watababe H, Kojima Y. Loxoprofen sodium treatment for elderly men with refractory nocturia: effect on night-time urine production. Int J Urol 2008;15:462-4.

8. Falahatkar S, Mokhtari G, Poureza F, Asgari SA, Kamran AN. Celecoxib for treatment of nocturia caused by benign prostatic hyperplasia: a prospective, randomized, double-blind, placebo-controlled study. Urology 2008;72:813-6.

9. Brubaker L, FitzGerald MP. Nocturnal polyuria and nocturia relief in patients treated with solifenacin for overactive bladder symptoms. Int Urogynecol J 2007; 18:737-41.

10. Fitzgerald MP, Lemack G, Wheeler T. Nocturia, nocturnal incontinence prevalence, and response to anticholinergic and behavioral therapy. Int Urogynecol J 2008;19:1545-50.

11. FitzGerald MP, Litman HJ, Link CL, McKinlay JB. The association of nocturia with cardiac disease, diabetes, body mass index, age and diuretic use: results from the Boston Area Community Health (BACH) survey. J Urol 2007;177:1385-9.

12. Coyne KS, Zhou Z, Battacharyya SK, Thompson CL, Dhawan R, Versi E. The prevalence of nocturia and its effect on health related quality of life and sleep in a community sample in the USA. BJU Int 2003;92:948-54.

13. Bing MH, Moller LA, Jennum P, Mortensen S, Skovgaard LT, Lose G. Prevalence and bother of nocturia, and causes of sleep interruption in a Danish population of men and women aged 60-80 years. BJU Int 2006;98:599-604.

14. Stewart RB, Moore MT, M ay FE, Marks RG, Hale WE. Nocturia: a risk factor for falls in the elderly. $J$ 
Am Geriatr Soc 1992;40:1217-20.

15. Pressman MR, Figueroa WG, Kendrick-Mohamed J, Greenspon LW, Peterson DD. Nocturia. A rarely recognized symptom of sleep apnea and other occult sleep disorders. Arch Intern Med 1996;156:545-50.

16. Asplund R, Aberg H. Diurnal variation in the levels of antidiuretic hormone in the elderly. J Intern Med 1991;229:131-4.

17. Kirkland JL, Lye M, Levy DW, Banerjee AK. Patterns of urine flow and electrolyte excretion in healthy elderly people. $\mathrm{Br}$ Med $J$ 1983;287:1665?7.

18. Jünemann KP, Hessdörfer E, Unamba-Oparah I, Berse $\mathrm{M}$, Brünjes $\mathrm{R}$, Madersbacher $\mathrm{H}$, et al. Propiverine hydrochloride immediate and extended release: comparison of efficacy and tolerability in patients with overactive bladder. Urol Int 2006;77:334-9.

19. Stichtenoth DO, Frolich JC. COX-2 and the kidneys. Curr Pharm Des 2000;6:173-5. 